EPJ Web of Conferences 49, 16002 (2013)

DOI: $10.1051 /$ epjconf/20134916002

(C) Owned by the authors, published by EDP Sciences, 2013

\title{
Studies of soft QCD at LHCb
}

\author{
Andrea Contu ${ }^{1, a}$, on behalf of the LHCb collaboration \\ ${ }^{1}$ INFN, Sezione di Cagliari, S. P. per Sestu Km 0.700, 09042 Monserrato (CA), Italy
}

\begin{abstract}
This paper summarises two analyses of minimum bias data collected with the LHCb detector from $p p$ collisions at the LHC. The geometry, tracking and particle identification system of the LHCb experiment provide unique capabilities for this type of study in a kinematic region where QCD models have large uncertainties. Measurements of prompt hadron production ratios and forward energy flow are presented and compared to predictions from MC event generators.
\end{abstract}

\section{Introduction}

The Large Hadron Collider Beauty experiment (LHCb) at CERN is a single-arm forward spectrometer designed for precision measurements of $\mathrm{CP}$ violation and rare $\mathrm{B}$ meson decays. It also allows the study of QCD processes in the forward region. The tracking system is composed of a precision Vertex Locator (VELO) surrounding the interaction region, a dipole magnet and three downstream tracking stations [1]. The resolution for primary (secondary) vertices is $\sim 50(100) \mu \mathrm{m}$ along the beam axis and the momentum resolution for tracks having hits in both the VELO and the tracking stations is around $0.5 \%$. Two Ring Imaging Cherenkov(RICH) detectors with three radiators provide excellent charged particle identification capabilities over a wide momentum range of $2-100 \mathrm{GeV} / c$. The results presented here are based on data collected during the 2010 LHC run at centre of mass energies of $\sqrt{s}=0.9 \mathrm{TeV}$ and $\sqrt{s}=7 \mathrm{TeV}$. The data were recorded using minimum bias (MB) triggers, which require a minimum energy deposit in the LHCb calorimeters or at least one reconstructed track in the event. Due to the finite beam size and crossing angle of the beams, the two halves of the VELO were partially retracted during the $\sqrt{s}=0.9 \mathrm{TeV}$ data taking. The partial closure of the VELO around the beam resulted in a non uniform detector acceptance around the beam axis and a slightly lower reconstruction efficiency for charged particles, particularly in the high pseudorapidity region. The measurements presented in this paper are the prompt hadron production ratios and the forward energy flow.

\section{Prompt hadron production ratios}

The measurement of the $\bar{p} / p$ production cross-section ratio is an important probe for baryon number transport (BNT) mechanisms. This ratio, together with other prompt hadron production ratios such as $K^{-} / K^{+}, \pi^{-} / \pi^{+},(p+$

\footnotetext{
a e-mail: Andrea.Contu@ cern.ch
}

$\bar{p}) /\left(K^{+}+K^{-}\right),(p+\bar{p}) /\left(\pi^{+}+\pi^{-}\right)$and $\left(K^{+}+K^{-}\right) /\left(\pi^{+}+\pi^{-}\right)$, provides information on the hadronisation mechanisms and is a valuable input to re-tunings of Monte Carlo (MC) event generators. The above ratios have been measured by the LHCb collaboration [2] at two $p p$ centre of mass energies as a function of pseudorapidity, $\eta$, in three bins of transverse momentum, $p_{T}$, and compared to several PYTHIA tunes [3-5]. Simulations are used to correct for non-prompt contamination, detector acceptance, reconstruction and tracking efficiency. Different particle species are selected using information from the RICH detectors. The PID efficiencies and misidentification rates are extracted from data using dedicated calibration samples of $\Lambda \rightarrow p \pi^{-}, \phi \rightarrow K^{+} K^{-}$and $K_{S}^{0} \rightarrow \pi^{+} \pi^{-}$. The PID performance is the major source of systematic uncertainty, also due to the limited size of the calibration samples. Other significant sources of systematic uncertainty are the knowledge and the implementation of the interaction cross-section of protons, kaons and pion with the detector material and the detector description in simulations.

The results for the $\bar{p} / p$ ratio are shown in Figure 1 . While the Perugia NOCR tune gives a good description of the data at $\sqrt{s}=0.9 \mathrm{TeV}$, the LHCbMC and Perugia0 tunes tend to underestimate the ratio. At $\sqrt{s}=7 \mathrm{TeV}$, the $\mathrm{LHCb}$ and Perugia0 tunes reproduce the data reasonably well, but the Perugia NOCR tune underestimate the ratio. This behaviour has been already observed in the $\bar{\Lambda} / \Lambda$ measurement performed by $\mathrm{LHCb}[6]$. The $\bar{p} / p$ results are summarised as a function of the rapidity loss, $\Delta y^{1}$, at both centre of mass energy tested, as shown in Figure 2. Within the Regge model, baryon production at high energy is driven by Pomeron exchange and baryon number transport by string-junction exchange [7]. Assuming this picture the $\Delta y$ dependence of the $\bar{p} / p$ ratio approximately

\footnotetext{
${ }^{1} \Delta y$ is defined as the difference $y_{\text {beam }}-y_{p(\bar{p})}$, where $y_{\text {beam }}$ is the rapidity of the incoming protons in the beam and $y_{p(\bar{p})}$ is the rapidity of the outgoing proton (antiproton)
} 

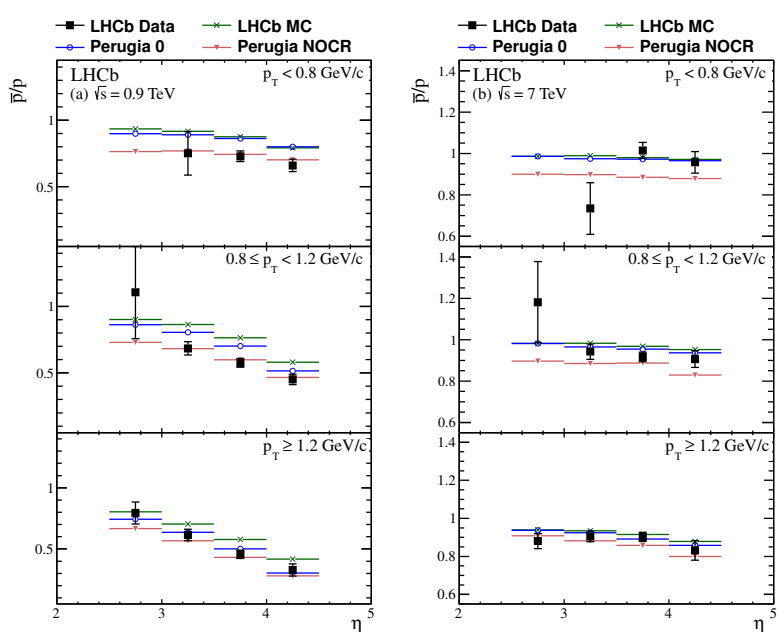

Figure 1. $\bar{p} / p$ ratio as a function of pseudorapidity at (a) $\sqrt{s}=$ $0.9 \mathrm{TeV}$ and (b) $\sqrt{s}=0.9 \mathrm{TeV}$.

follows the form

$$
\frac{\bar{p}}{p}=\frac{1}{1+C \exp \left[\left(\alpha_{J}-\alpha_{P}\right) \Delta y\right]},
$$

where $C$ determines the relative contributions of the two mechanisms, and $\alpha_{J}\left(\alpha_{P}\right)$ is the intercept of the string junction (Pomeron) Regge trajectory. A fit has been performed using LHCb and ALICE [8] measurements, with both $C$ and $\left(\alpha_{J}-\alpha_{P}\right)$ left as free parameters and determined to be $22.5 \pm 6.0$ and $-0.98 \pm 0.07$ respectively, with a $\chi^{2} / \mathrm{ndf}$ of 8.7/8. Taking $\alpha_{P}=1.2 \quad$ [9] suggests a low value of $\alpha_{J}$, significantly below the $\alpha_{J} \approx 0.5$ expected if the stringjunction intercept is associated with that of the standard Reggeon (or meson). The measurements of the $K^{-} / K^{+}$

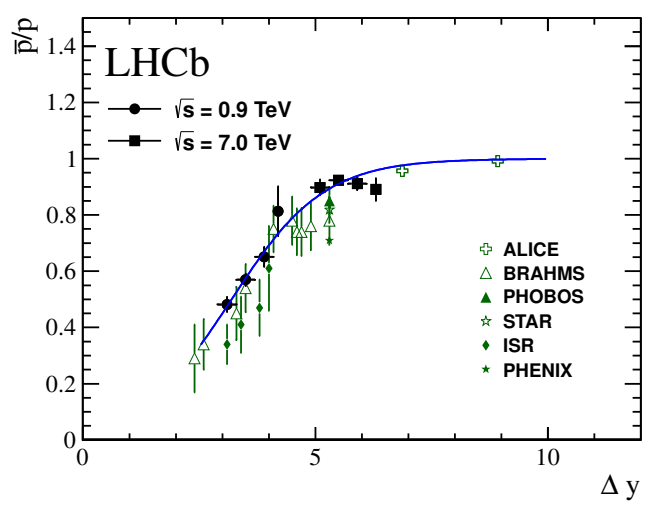

Figure 2. LHCb results for $\bar{p} / p$ as a function of the rapidityloss, $\Delta y$. Measurements from other experiments are superimposed. The blue line represents the fit to ALICE and LHCb data, as described in the text.

and $\pi^{-} / \pi^{+}$ratios are plotted in Figure 3 and Figure 4 respectively. Small discrepancies are observed at $7 \mathrm{TeV}$ for the former at low $p_{T}$, and the latter at high $p_{T}$. Similarly to the $\bar{p} / p$ ratio, the $K^{-} / K^{+}$and $\pi^{-} / \pi^{+}$ratios also differ from unity, most noticeably towards high $p_{T}$ and high $\eta$. This behaviour is in general well modelled by all the generator tunes. When comparing the measurements and predic-
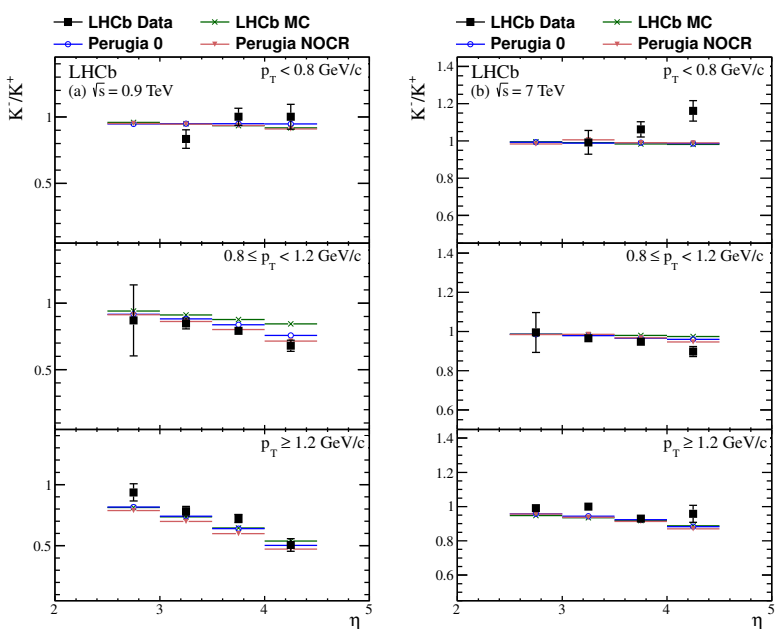

Figure 3. $K^{-} / K^{+}$ratio as a function of pseudorapidity at (a) $\sqrt{s}=0.9 \mathrm{TeV}$ and (b) $\sqrt{s}=0.9 \mathrm{TeV}$.
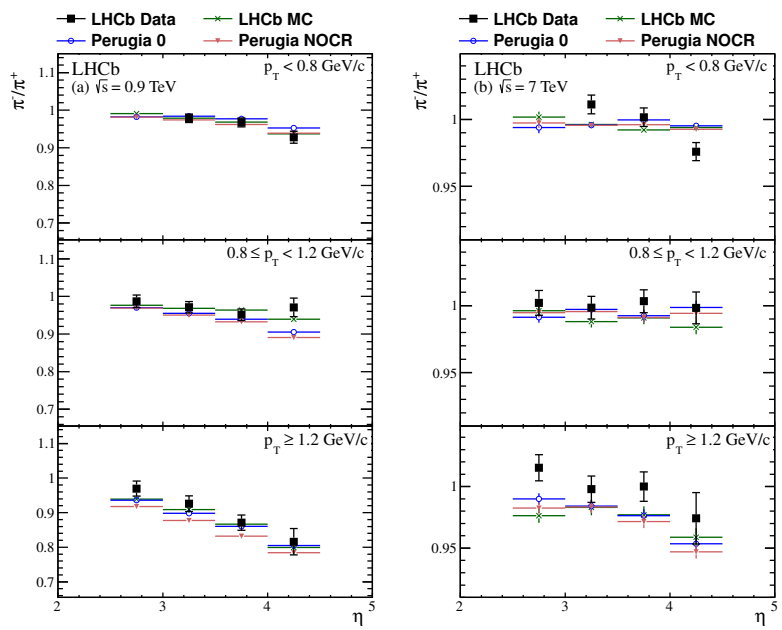

Figure 4. $\pi^{-} / \pi^{+}$ratio as a function of pseudorapidity at (a) $\sqrt{s}=$ $0.9 \mathrm{TeV}$ and (b) $\sqrt{s}=0.9 \mathrm{TeV}$.

tions for the different-particle ratios the most striking differences occur for $(p+\bar{p}) /\left(\pi^{+}+\pi^{-}\right)$, shown in Figure 5, and $\left(K^{+}+K^{-}\right) /\left(\pi^{+}+\pi^{-}\right)$, shown in Figure 6. The Perugia0 and Perugia NOCR tunes underestimate the ratios. On the other hand, the LHCbMC gives a significantly different prediction which is in reasonable agreement with the data. The $(p+\bar{p}) /\left(\pi^{+}+\pi^{-}\right)$result is consistent with an overestimation of the baryon over meson suppression, as observed in another LHCb measurement, the $\bar{\Lambda} / K_{S}^{0}$ ratio [6]. Finally, the $(p+\bar{p}) /\left(K^{+}+K^{-}\right)$shown in Figure 7, although of difficult theoretical interpretation, is in good agreement with PYTHIA predictions. 

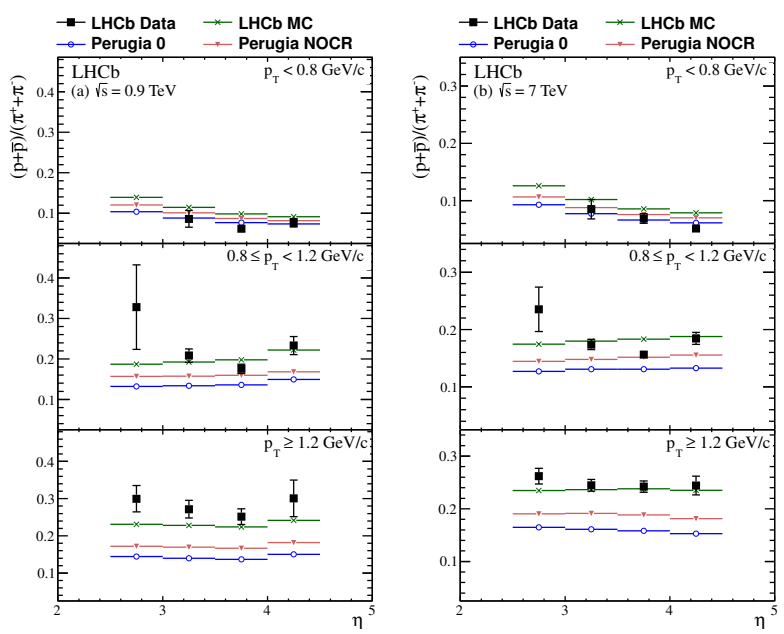

Figure 5. $(p+\bar{p}) /\left(\pi^{+}+\pi^{-}\right)$ratio as a function of pseudorapidity at (a) $\sqrt{s}=0.9 \mathrm{TeV}$ and (b) $\sqrt{s}=0.9 \mathrm{TeV}$.
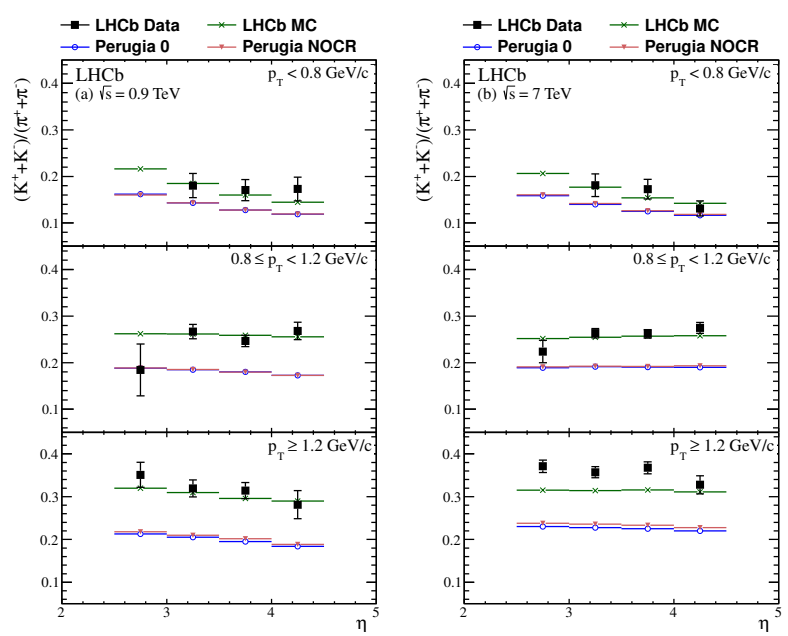

Figure 6. $\left(K^{+}+K^{-}\right) /\left(\pi^{+}+\pi^{-}\right)$ratio as a function of pseudorapidity at (a) $\sqrt{s}=0.9 \mathrm{TeV}$ and (b) $\sqrt{s}=0.9 \mathrm{TeV}$.

\section{Forward energy flow}

The measurement of the forward energy flow is a probe for parton radiation and multi-parton interaction (MPI). MPI becomes increasingly important at LHC collision energies, where inelastic interactions between very soft partons are sufficiently energetic to contribute to final state particle production. Moreover, a measurement provides an excellent test for collision physics and cosmic ray models. Theoretically, the energy flow is defined as

$$
E F_{t h}=\frac{1}{N_{i n t}} \frac{d E_{t o t}}{d \eta}
$$

where, $N_{\text {int }}$ is the number of inelastic $p p$ collisions and $d E_{t o t}$ is the energy flowing in pseudorapidity of $d \eta$. Experimentally, the measurement is defined in bins of pseu-
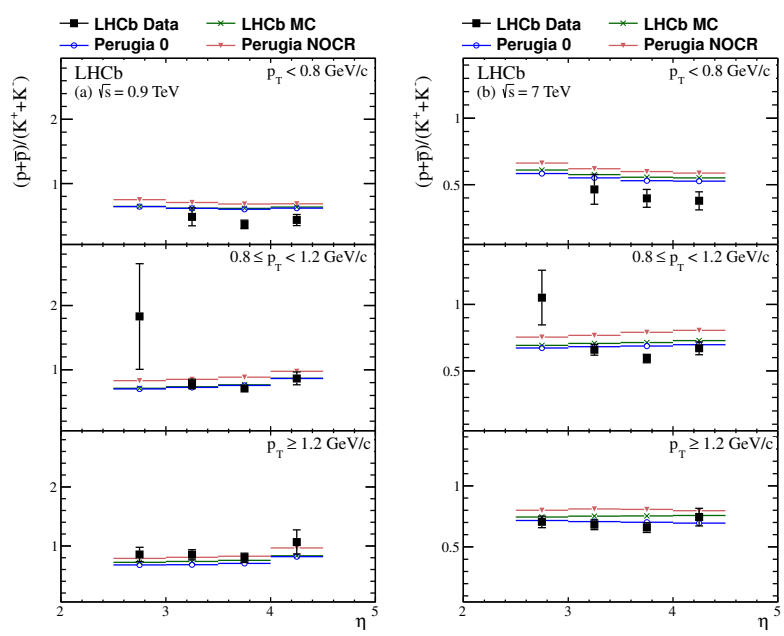

Figure 7. $(p+\bar{p}) /\left(K^{+}+K^{-}\right)$ratio as a function of pseudorapidity at (a) $\sqrt{s}=0.9 \mathrm{TeV}$ and (b) $\sqrt{s}=0.9 \mathrm{TeV}$.

dorapidity:

$$
E F=\frac{1}{\Delta \eta}\left(\frac{1}{N_{\text {int }}} \sum_{i=1}^{N_{\text {part }, \eta}} E_{i, \eta}\right) .
$$

LHCb measured the charged and total energy flow using $0.1 \mathrm{nb}^{-1}$ of collision data collected by $\mathrm{LHCb}$ during a low luminosity run at $\sqrt{s}=7 \mathrm{TeV}$ [10]. The measurement is performed in the region $1.9<\eta<4.9$ as a function of pseudorapidity and compared to several PYTHIA tunes $[4,5,11]$ and cosmic ray models [12-14]. In order to maximise the information that can be extracted, the data sample is subdivided in four event categories:

- Inclusive MB: at least one reconstructed track with momentum $p>2 \mathrm{GeV} / c$;

- Hard scattering: at least one reconstructed track with $p_{T}>3 \mathrm{GeV} / c$;

- Diffractive enriched: inclusive MB events with no tracks in the region $-3.5<\eta<-1.5$;

- Non-diffractive enriched: inclusive MB events with at least one track in the region $-3.5<\eta<-1.5$.

The division above is possible thanks to the acceptance of the VELO in the backward region.

The energy flow carried by charged particles (charged energy flow) is the primary measurement and is performed using only tracking information and neglecting the particle masses. The total energy flow is determined using dataconstrained MC to estimate the neutral component based on information from the electromagnetic calorimeter. Systematic uncertainties include tracking efficiencies differences in data and simulation, pile-up contributions and the model dependence of the correction factor which correct the measured energy flow for detector effects.

Results for the charged energy flow are shown in Figures 8 and 9 compared to PYTHIA tunes and cosmic ray models respectively. It is noted that the charged energy flow rises more steeply at high $\eta$ than predicted 
by PYTHIA 6 tunes while the low $\eta$ region is generally well described, except for the diffractive enriched category. In this event sample, a good description over the entire $\eta$ range is given by PYTHIA 8 . The measure-
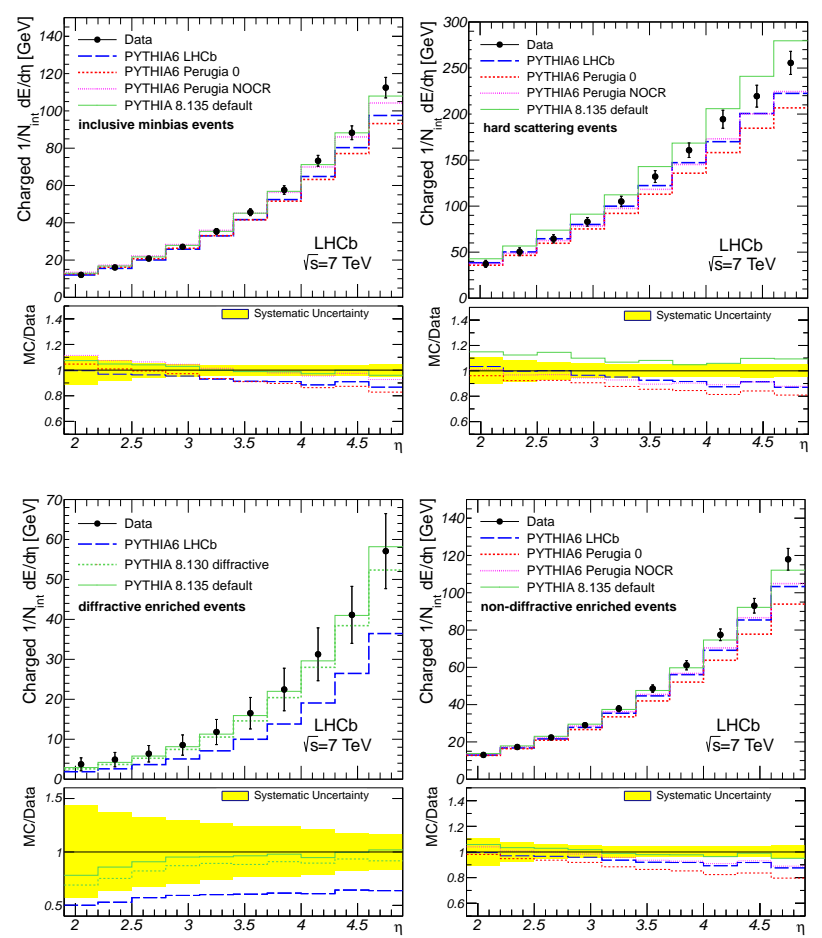

Figure 8. Charged energy flow in bins of $\eta$ for the four categories compared to several PYTHIA tunes.

ments performed with inclusive minimum-bias and nondiffractive enriched events are well described by the EPOS and SIBYLL models, while both QGSJET versions overestimate the charged energy flow. The latter also occurs at large $\eta$ in the case of hard scattering interactions for all cosmic-ray interaction models, except the QGSJETII03. The diffractive enriched charged energy flow is underestimated by all cosmic-ray interaction models.

Similar conclusions can be drawn for the total energy flow, shown in Figures 10 and 11. It can be clearly seen that all PYTHIA 6 tunes underestimate the total energy flow at large pseudorapidity for all event classes. The PYTHIA 8 generator gives the best description of the measurements performed with inclusive minimum-bias, diffractive and non-diffractive enriched events categories, except for the pseudorapidity range $1.9<\eta<2.5$. The hard scattering category is not accurately described by any of these models. The total energy flow is well modelled by the SIBYLL and EPOS generators for the inclusive minimum bias and non-diffractive enriched categories. In the case of hard scattering, the best description of the data at large $\eta$ is given by the QGSJETII-03 generator. The total energy flow in the diffractive enriched category is underestimated at large $\eta$ by all cosmic ray interaction models.
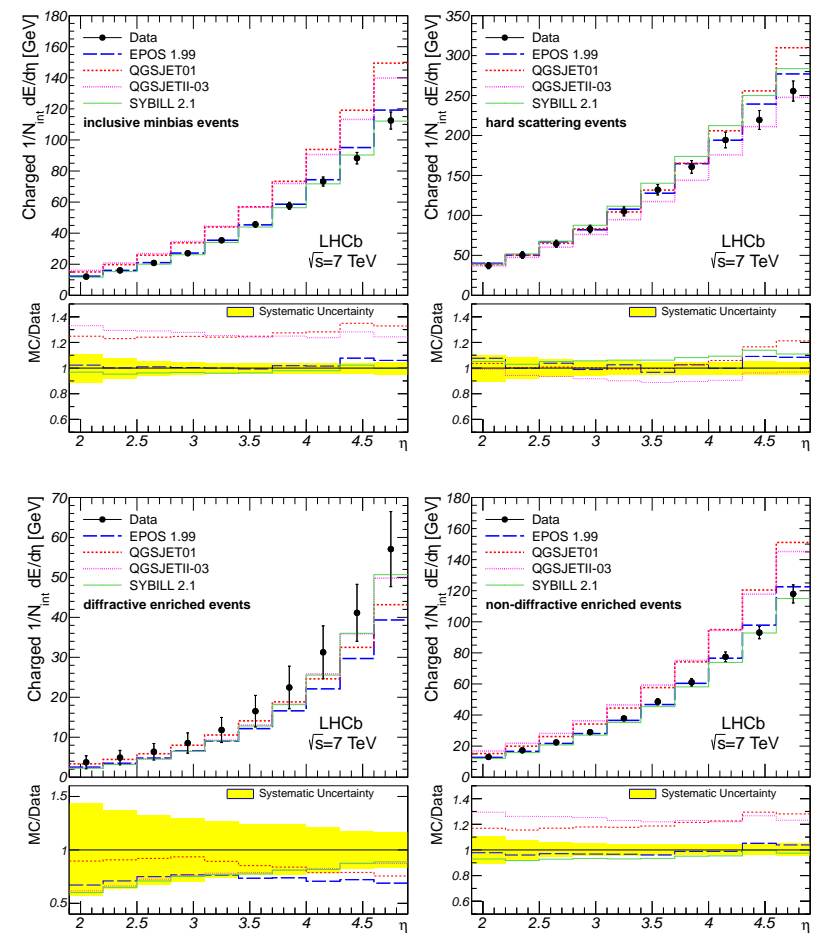

Figure 9. Charged energy flow in bins of $\eta$ for the four categories compared to cosmic ray model predictions.
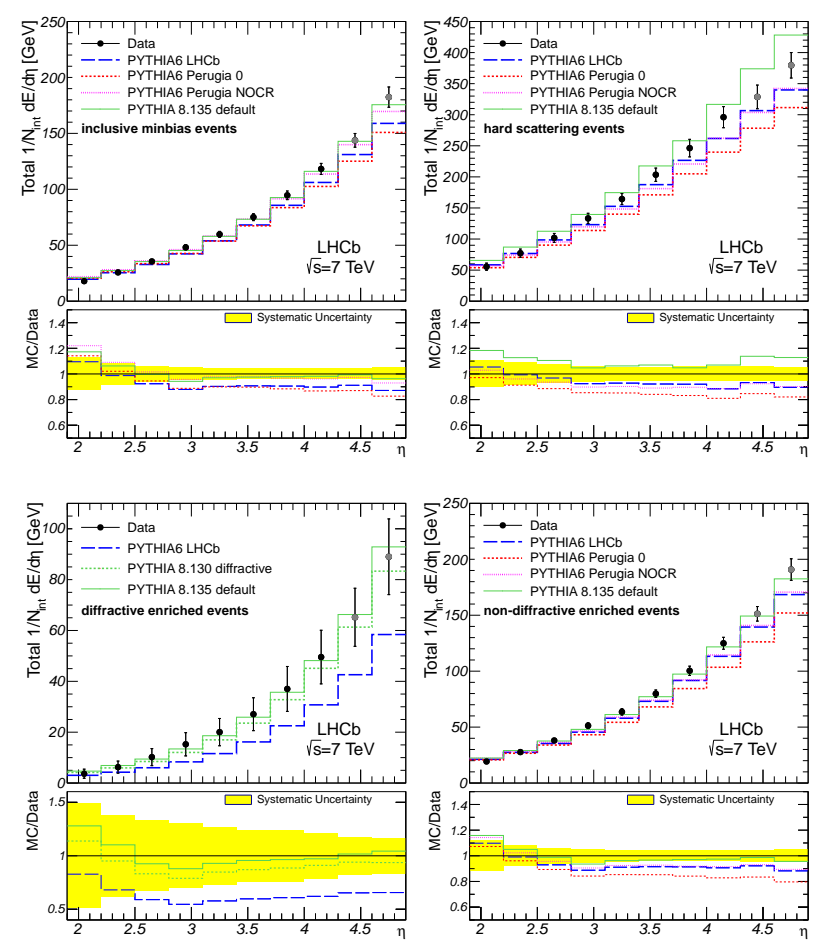

Figure 10. Total energy flow in bins of $\eta$ for the four categories compared to several PYTHIA tunes.

\section{Conclusions}

$\mathrm{LHCb}$ provides an excellent environment for performing soft-QCD studies at high $\eta$ and low $p_{T}$. The two most 

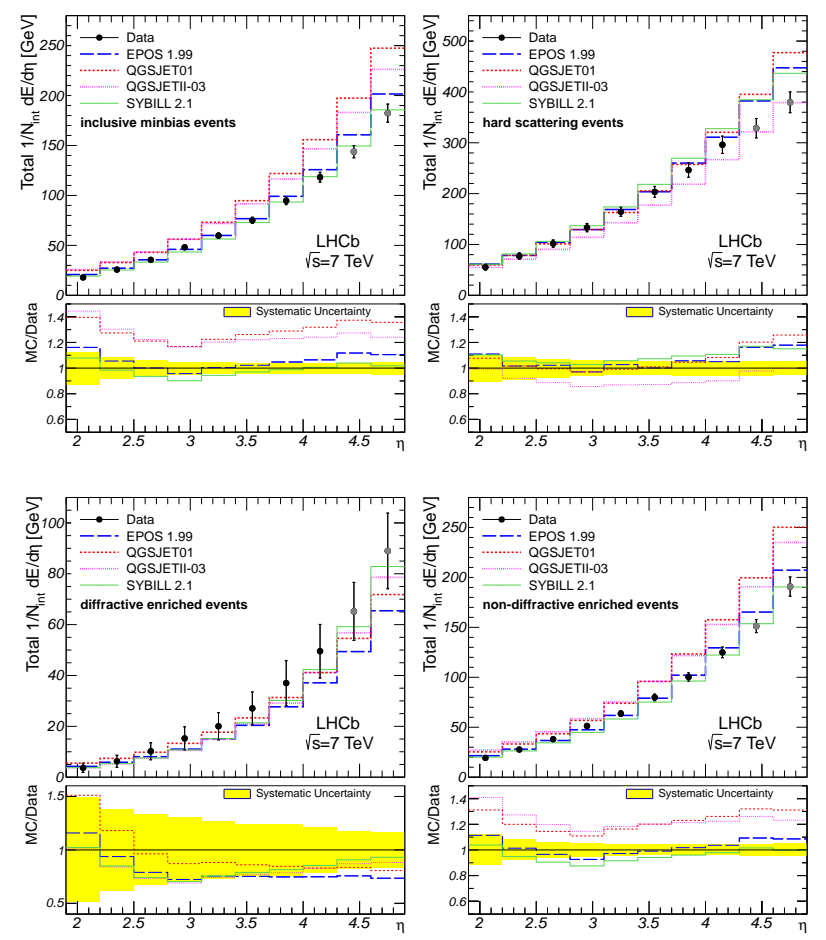

Figure 11. Total energy flow in bins of $\eta$ for the four categories compared to cosmic ray model predictions.

recent $\mathrm{LHCb}$ results in this area have been presented in this document.

Measurements of prompt hadron production ratios have been performed using data recorded at $\sqrt{s}=0.9$ and $7 \mathrm{TeV}$. It is the first time such measurements are performed at the latter centre of mass energy. No single
PYTHIA 6 tune is able to describe all the observables. The baryon transport has been studied using the $\bar{p} / p$ ratio which provides a precise measurement over the largest $\Delta y$ range covered by a single experiment.

None of the compared generators is able to consistently describe the energy flow correctly for all the event categories studied. In general, PYTHIA 6 tunes underestimate the energy flow at high $\eta$. On the other hand, cosmic ray generators have a tendency to overestimate the energy flow.

\section{References}

[1] The LHCb Collaboration, JINST 3, (2008) S08005.

[2] The LHCb Collaboration, EPJC 72, (2012) 2168.

[3] T. Sjöstrand, S. Mrenna and P. Skands, JHEP 05 (2006) 026.

[4] I. Belyaev et al., IEEE Nucl. Sci. Symp. Conf. Rec. (2010) 1155.

[5] P. Z. Skands, Phys. Rev. D 82, (Oct, 2010) 074018.

[6] The LHCb Collaboration, JHEP 1108, (2011) 34.

[7] D. Kharzeev, Phys. Lett. B 378 (1996) 238.

[8] The ALICE Collaboration, Phys. Rev. Lett., 105 072002, (2010).

[9] A. B. Kaidalov, L. A. Ponomarev, and K. A. TerMartirosyan, Sov. J. Nucl. Phys. 44, (1986) 468.

[10] The LHCb Collaboration, arXiv:1212.4755.

[11] T. Sjöstrand, S. Mrenna, P. Skands, Comput. Phys. Commun. 178 850, (2008).

[12] T. Pierog and K. Werner, Nucl. Phys. Proc. Suppl. 196, (2009) 102.

[13] S. Ostapchenko, AIP Conf. Proc. 928, (2007) 118.

[14] E.-J. Ahn et al., Phys. Rev. D80, (2009) 094003. 